\title{
CORD BLOOD H- AND L-FICOLIN CONCENTRATIONS IN TERM PREGNANCIES WITH NORMAL AND RESTRICTED FETAL GROWTH
}

\author{
S. Liosi ${ }^{1}$, T. Boutsikou ${ }^{1}$, M. Boutsikou ${ }^{1}$, D.D. Briana ${ }^{1}$, L. Stamati ${ }^{2}$, S. Baka ${ }^{1}$, D. Hassiakos ${ }^{1}$, D. Gourgiotis ${ }^{2}$, \\ A. Malamitsi-Puchner ${ }^{1}$ \\ ${ }^{1}$ Neonatal Division, 2nd Department of Obstetrics and Gynecology, ${ }^{2}$ Research Laboratories, 2nd \\ Department of Pediatrics, Athens University Medical School, Athens, Greece
}

Background and aims: Ficolins $H$ and $L$ are serum defence proteins, mainly expressed in the liver and differing in ligand specificity. They initiate the lectin pathway of complement activation through association with mannose-binding-protein- associated proteases (MASPs). Ficolins are implicated in the pathogenesis of infectious and autoimmune diseases, apoptosis and preeclampsia. H-ficolin is a powerful direct opsonin binding to late apoptotic cells. This study aimed to determine $\mathrm{H}$ - and L-ficolin cord blood concentrations in appropriate-for-gestational-age (AGA) and intrauterine-growth-restricted pregnancies (IUGR), associated with excessive apoptosis, uteroplacental vascular insufficiency and activation of the innate immune system. Furthermore to investigate possible correlations of $\mathrm{H}$ - and L-ficolin concentrations with several demographic parameters of infants at birth.

Methods: Serum H- and L-ficolin concentrations were determined by ELISA in 160 mixed arteriovenous cord blood samples from 50 IUGR and 110 AGA singleton full-term pregnancies.

Results: No significant differences in cord blood H- and L-ficolin concentrations were observed between IUGR and AGA groups. The effect of customized centile, birthweight, gender, gestational age, parity and maternal age on cord blood $\mathrm{H}$ - and L-ficolin concentrations was not significant in both groups.

Conclusions: Lack of differences in cord blood H- and L- ficolin concentrations at term between IUGR and AGA groups possibly suggests that they are not directly implicated in the pathophysiological processes of IUGR. Possibly this happens because the lectin pathway of complement activation, as far as ficolins are concerned, may not be fully functional at birth. 